\title{
Natural Course and Risk of Cholangiocarcinoma in Patients with Recurrent Pyogenic Cholangitis: A Retrospective Cohort Study
}

\author{
Min su You ${ }^{1}$, Sang Hyub Lee ${ }^{1}$, Jinwoo Kang ${ }^{1}$, Young Hoon Choi ${ }^{1}$, Jin Ho Choi ${ }^{1}$, Bang-sup Shin ${ }^{1}$, Gunn Huh ${ }^{1}$, Woo Hyun \\ Paik $^{1}$, Ji Kon Ryu ${ }^{1}$, Yong-Tae Kim ${ }^{1}$, Dong Kee Jang ${ }^{2}$, and Jun Kyu Lee \\ ${ }^{1}$ Department of Internal Medicine and Liver Research Institute, Seoul National University College of Medicine, Seoul, and ${ }^{2}$ Department of \\ Internal Medicine, Dongguk University Ilsan Hospital, Goyang, Korea
}

Background/Aims: Recurrent pyogenic cholangitis (RPC) is a chronic progressive disease frequently accompanied by cholangiocarcinoma (CCA). This study aimed to investigate the natural course of RPC and identify factors associated with CCA. Methods: From January 2005 to December 2016, 310 patients diagnosed with RPC at Seoul National University Hospital were included. Complications and management during follow-up were recorded. CCA-free probability was estimated by Kaplan-Meier method, and risk factors associated with CCA were analyzed using log-rank test and Cox's proportional hazard regression model. Results: Mean age at diagnosis was $59.1 \pm 10.9$ years and mean follow-up duration was $84.0 \pm 64.1$ months. An intrahepatic duct stone was found in 253 patients (81.6\%). Liver atrophy was identified in 185 patients (59.7\%) and most commonly located at the left lobe (65.4\%). Acute cholangitis, liver abscesses, cirrhotic complications, and CCA developed in 41.3\%, 19.4\%, 9.7\%, and $7.4 \%$, respectively. During follow-up, complete resolution rate after hepatectomy, biliary bypass surgery, and choledocholithotomy with T-tube insertion reached $82.3 \%$, $55.2 \%$, and $42.1 \%$, respectively. None of the patients who maintained complete resolution by the last follow-up day developed CCA. In univariate analysis, female, both-sided intrahepatic duct stones, and liver atrophy at any location were associated with increased risk of CCA. Multivariate analysis revealed that both-sided atrophy significantly increased risk of CCA (hazard ratio, 4.56; 95\% confidence interval, 1.48 to 14.09; $p=0.008)$. In 21 patients who developed intrahepatic CCA, tumor was located mostly in the atrophied lobe $(p=0.023)$. Conclusions: In RPC patients, acute cholangitis, liver abscess, cirrhotic complications, and CCA frequently developed. Both-sided liver atrophy was a significant risk factor for developing CCA. (Gut Liver 2019;13:373-379)

Key Words: Cholangitis; Prognosis; Atrophy; Cirrhosis; Cholangiocarcinoma

\section{INTRODUCTION}

Recurrent pyogenic cholangitis (RPC) is typified by de novo intrahepatic duct (IHD) stones, bile duct strictures or dilatation, and recurrent attacks of cholangitis. It was first described by Digby ${ }^{1}$ and named RPC in $1954 .^{2}$ RPC is also referred to as "Hong Kong disease," "Oriental cholangiohepatitis," "Oriental infestational cholangitis," and "biliary obstruction syndrome of the Chinese," suggesting that an incidence varies according to geographical location, occurring mostly in Asia. ${ }^{3}$ However, as internationalization has progressed, understanding of the disease is also important in Western countries.

RPC can be diagnosed when there are IHD stones, bile duct strictures or dilatation, and liver atrophy, accompanied by recurrent symptoms indicative of cholangitis, including fever, abdominal pain, and jaundice. ${ }^{4}$ During its chronic course, multiple RPC-related complications can occur, such as acute cholangitis, liver abscesses, cirrhosis, and cholangiocarcinoma (CCA). ${ }^{5,6}$ Although RPC is a progressive disease that causes the variety of complications, ${ }^{4,5}$ there have been few studies regarding the natural course of RPC based on long-term follow-up data. In addition, although RPC is known to increase the risk of CCA, there are insufficient data to determine factors associated with CCA.

To manage and prevent long-term complications, including CCA, patients with RPC often need several surgical interventions during its course. Hepatic resection, bypass surgery, and liver transplantation can be considered surgical treatment modalities

Correspondence to: Sang Hyub Lee (https://orcid.org/0000-0003-2174-9726)

Department of Internal Medicine and Liver Research Institute, Seoul National University Hospital, Seoul National University College of Medicine, 101 Daehak-ro, Jongno-gu, Seoul 03080, Korea

Tel: +82-2-2072-2228, Fax: +82-2-762-9662, E-mail: gidoctor@snuh.org

Received on July 31, 2018. Revised on September 14, 2018. Accepted on October 2, 2018. Published online March 15, 2019 pISSN 1976-2283 eISSN 2005-1212 https://doi.org/10.5009/gnl18339

@ This is an Open Access article distributed under the terms of the Creative Commons Attribution Non-Commercial License (http://creativecommons.org/licenses/by-nc/4.0) which permits unrestricted non-commercial use, distribution, and reproduction in any medium, provided the original work is properly cited. 
for RPC. ${ }^{7}$ Hepatic resection is most widely used, and complete remission can be achieved in $64.0 \%$ to $84.4 \%$ of patients. However, postoperative complications, such as wound infection, bile leak, hernia, and multiorgan failure, occur in $17.5 \%$ to $40 \%$ of these patients, ${ }^{4,7}$ emphasizing the need for thorough risk stratification and adequate patient selection.

It is essential to understand RPC-related complications and identify high-risk patients who may need early surgical intervention. Therefore, this study aimed to investigate the natural course of RPC and identify factors related to CCA.

\section{MATERIALS AND METHODS}

\section{Study subjects}

This study enrolled patients with RPC who visited Seoul National University Hospital from January 2005 to December 2016. Characteristic imaging findings of RPC included IHD stones, strictures, and dilatation with or without liver atrophy based on ultrasonography (US), computed tomography (CT), and magnetic resonance imaging (MRI). Among 585 patients who were diagnosed with RPC based on typical imaging findings reported by specialty radiologists, ${ }^{4,9} 366$ patients with a follow-up duration of at least 6 months were analyzed.

As an exclusion criterion, primary sclerosing cholangitis (PSC) was diagnosed based on laboratory findings and specific imaging findings, such as diffuse IHD strictures in combination with colonoscopic findings suggestive of ulcerative colitis. The diagnosis of CCA, intraductal papillary neoplasms of the bile duct, hepatocellular carcinoma, and desmoid tumor was made by imaging tests, laboratory and/or pathological findings. ${ }^{10}$ After exclusion of 35 patients with an initial presentation of CCA, 14 with choledochal cysts, three with PSC, and four with biliary strictures caused by other tumors, a total of 310 patients were enrolled (Fig. 1). This study was approved by Institutional Review Board of the Seoul National University Hospital, Seoul, Korea (IRB number: 1703-046-837). All patients provided written informed consent before any intervention and surgical treatment.

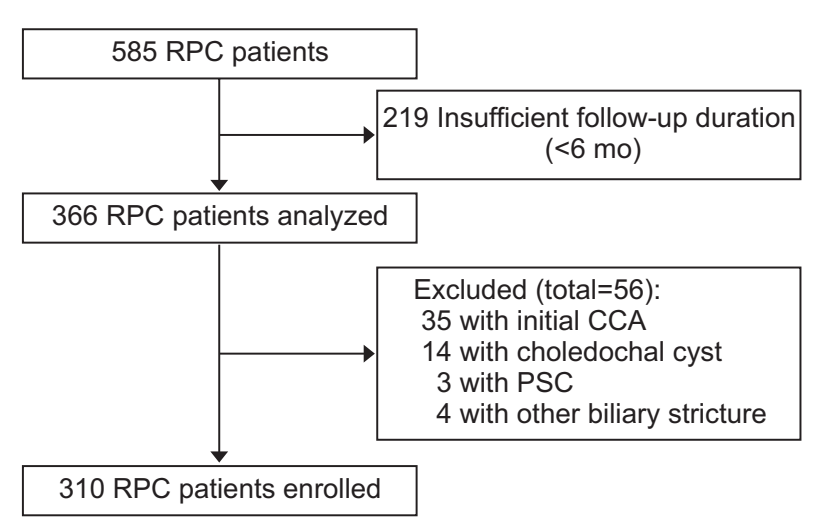

Fig. 1. Flowchart of patient enrollment.

RPC, recurrent pyogenic cholangitis; CCA, cholangiocarcinoma; PSC, primary sclerosing cholangitis.

\section{Data analysis and definition of events}

At the time of initial enrollment, patient demographics, previous surgical history, imaging findings regarding the distribution of biliary stones, bile duct strictures or dilatation, and liver atrophy were identified. Asymptomatic patients without bile duct stones were followed at regular intervals of 6 to 12 months, and CT and/or US were used as follow-up imaging tools. In patients with a history of bile duct stones or acute cholangitis, complete blood count and liver function tests were conducted at intervals of 3 to 6 months in addition to the imaging examinations. During follow-up, the occurrence of RPC-related complications, including acute cholangitis, liver abscesses, cirrhotic complications, and CCA, were monitored based on the patient's symptoms and findings of physical, imaging and laboratory examinations. The follow-up period was defined as the time from the first diagnosis to the last date of imaging tests covering the liver, gallbladder, and biliary tract.

RPC-related biliary cirrhosis was defined as liver cirrhosis caused by RPC in the absence of viral and/or autoimmune hepatitis. Cirrhotic complications were defined as varices, ascites, and portosystemic encephalopathy related to biliary cirrhosis with portal hypertension. ${ }^{11}$ Liver abscesses were diagnosed based on imaging findings of often multiple, peripherally enhanced, and centrally hypoattenuated lesions associated with increased serum inflammatory marker levels. ${ }^{12}$ All patients with CCA were diagnosed by pathological confirmation.

Surgical treatment included hepatectomy, biliary bypass surgery (choledochojejunostomy and hepaticojejunostomy), choledocholithotomy with T-tube insertion, and liver transplantation. Liver resection was performed in patients with IHD stones together with intrahepatic bile duct strictures and liver atrophy. Biliary bypass surgery was carried out in patients with multiple IHD stones accompanied by extrahepatic bile duct stenosis in the absence of hepatic parenchymal involvement. Liver transplantation was conducted if diffuse involvement of IHD stones, RPCrelated atrophy, and cirrhosis were confirmed. Nonsurgical therapy included endoscopic retrograde cholangiopancreatography with stone removal, endoscopic retrograde biliary drainage, and percutaneous transhepatic cholangioscopic lithotripsy (PTCSL).

\section{Statistical analysis}

Continuous variables are presented as the mean \pm standard deviation, and categorical variables are summarized by frequencies and percentages. The Pearson chi-square test or Fisher exact test was used, where appropriate, for the comparison of categorical data to calculate the statistical significance of differences in the demographic and clinical variables. The CCA-free probability was estimated using the Kaplan-Meier method.

To determine the risk factors for CCA, baseline patient demographics, imaging findings, and complications during follow-up were evaluated. The hazard ratio (HR) for an increased risk of 
CCA and the 95\% confidence interval (CI) were calculated using the Cox proportional hazard regression model. Univariate and multivariate analyses were conducted to assess the association of various factors with the development of CCA. In the multivariate logistic regression analysis, probable risk factors for CCA were evaluated using Akaike Information Criterion-based backward selection. ${ }^{13}$ p-values $\leq 0.05$ were considered statistically significant in the final multivariate model. All statistical computations were performed in $\mathrm{R}$ version 3.3.3 (The R Foundation for Statistical Computing, Vienna, Austria).

\section{RESULTS}

\section{Clinical characteristics of patients}

The demographics, baseline imaging findings, and prior surgical history of the patients are summarized in Table 1 . The mean age was $59.1 \pm 10.9$ years, and 219 patients (70.6\%) were women. The initial diagnosis was based on a CT scan in 222 patients (71.6\%), US findings in 65 patients (21.0\%), and an MRI scan in 23 patients (7.4\%). An IHD stone was found in 253 patients

Table 1. Baseline Characteristics $(n=310)$

\begin{tabular}{|c|c|}
\hline Characteristic & Value \\
\hline Age, yr & $59.1 \pm 10.9$ \\
\hline \multicolumn{2}{|l|}{ Sex } \\
\hline Female & 219 (70.6) \\
\hline Male & $91(29.4)$ \\
\hline \multicolumn{2}{|l|}{ IHD stone } \\
\hline Both & $66(21.3)$ \\
\hline Left & $140(45.2)$ \\
\hline Right & $47(15.2)$ \\
\hline \multicolumn{2}{|l|}{ IHD dilatation or stricture } \\
\hline Both & $160(51.6)$ \\
\hline Left & 103 (33.2) \\
\hline Right & 39 (12.6) \\
\hline \multicolumn{2}{|l|}{ Liver atrophy } \\
\hline Both & $23(7.4)$ \\
\hline Left & $121(39.0)$ \\
\hline Right & $41(13.2)$ \\
\hline CBD stone & $122(39.4)$ \\
\hline CBD dilatation or stricture & $112(36.1)$ \\
\hline GB stone & $32(10.3)$ \\
\hline \multicolumn{2}{|l|}{ Prior surgery } \\
\hline Cholecystectomy & $129(41.6)$ \\
\hline Hepatectomy & $33(10.7)$ \\
\hline Biliary bypass surgery* & $30(9.7)$ \\
\hline Choledocholithotomy & $15(4.8)$ \\
\hline
\end{tabular}

Data are presented as mean \pm SD or number $(\%)$. IHD, intrahepatic duct; CBD, common bile duct; GB, gallbladder. *Includes choledochojejunostomy and hepaticojejunostomy.
(81.6\%), and liver atrophy was identified in 185 patients (59.7\%). The hepatic lobe most frequently affected by IHD stones and liver atrophy was the left lobe.

IHD dilatation and/or strictures were confirmed in 302 patients (97.4\%); in both lobes in 160 patients (51.6\%) and in the left lobe in 103 patients (33.2\%). A common bile duct (CBD) stone was identified in 122 patients (39.4\%) and was accompanied by CBD dilatation and/or strictures in 112 patients (36.1\%). The combined number of patients with a gallbladder stone and a previous history of cholecystectomy was 151 (51.9\%). Among 20 patients with "too many IHDs", a sign indicative of clonorchiasis, one patient was found to have stool containing Clonorchis sinensis eggs.

\section{Natural course and management during follow-up}

The mean follow-up duration was $84.0 \pm 64.1$ months. During follow-up, acute cholangitis requiring hospitalization occurred in 128 patients (41.3\%), and nonsurgical intervention was employed to remove bile duct stones and treat acute cholangitis; 115 patients (37.2\%) were treated with endoscopic retrograde cholangiopancreatography with stone removal, 65 patients (21.0\%) with endoscopic biliary stent insertion, and 53 patients (17.1\%) with PTCSL. Biliary cirrhosis developed in 51 patients (16.5\%); Child-Pugh A in 25 (49.0\%), Child-Pugh B in 18 (35.2\%), and Child-Pugh C in eight (15.7\%) patients. Other than biliary cirrhosis, the etiologies were viral hepatitis B (12/21, $57.1 \%)$, viral hepatitis C (5/21, 23.8\%), alcoholic hepatitis (3/21, $14.3 \%)$, and autoimmune hepatitis (1/21, 4.8\%). Among patients with biliary cirrhosis, portal hypertensive complications including varices, ascites, and portosystemic encephalopathy occurred in 30 patients (58.8\%). Table 2 summarizes the clinical course and management during follow-up.

Overall, CCA occurred in 23 patients (7.4\%), and the median duration from first diagnosis to CCA development was 49.9 months (interquartile range, 43.0 to 106.9 months). Of these, six patients (26.1\%) were eligible for curative resection. In 17 patients (73.9\%) who were diagnosed at an advanced stage, nine patients underwent conventional chemotherapy. The mean carcinoembryonic antigen (CEA) and carbohydrate antigen 19-9 (CA 19-9) levels at the time of CCA diagnosis were $10.7 \pm 15.6$ $\mathrm{ng} / \mathrm{mL}$ and 3,420.2 $\pm 7,858.0 \mathrm{U} / \mathrm{mL}$, respectively. The cumulative incidences of acute cholangitis, liver abscesses, cirrhotic complications and CCA are illustrated in Fig. 2.

Hepatectomy was the most frequently implemented surgical method, followed by biliary bypass surgery, choledocholithotomy with T-tube insertion, and liver transplantation (Table 2). Among 34 patients with bilateral IHD stones, only 14 patients (41.2\%) remained stone-free after surgery; 18 patients (52.9\%) were treated with left hepatic resection, five patients (14.7\%) with right hepatic resection, two patients (5.9\%) with bilateral resection, two patients (5.9\%) with liver transplantation, and seven patients (20.6\%) with choledocholithotomy and T-tube insertion.

The complete resolution rate of hepatectomy, biliary bypass 
Table 2. Clinical Course and Management during Follow-up ( $n=310)$

\begin{tabular}{lc}
\multicolumn{1}{c}{ Variable } & Value \\
\hline Complications & $128(41.3)$ \\
Acute cholangitis & $60(19.4)$ \\
Liver abscess & $51(16.5)$ \\
Biliary cirrhosis & $23(7.4)$ \\
Cholangiocarcinoma & \\
Surgical therapy & $115(37.1)$ \\
Hepatectomy & $98(31.6)$ \\
Left & $15(4.8)$ \\
Right & $2(0.6)$ \\
Both & $29(9.4)$ \\
Biliary bypass surgery* & $19(6.1)$ \\
Choledocholithomy & $4(1.3)$ \\
Liver transplantation & $65(21.0)$ \\
Non-surgical therapy & $53(17.1)$ \\
PTCSL & \\
ERCP with stone removal & \\
ERBD & \\
Follow-up duration, mo & \\
\hline
\end{tabular}

Data are presented as number (\%) or mean \pm SD.

PTCSL, percutaneous transhepatic cholangioscopic lithotripsy; ERCP, endoscopic retrograde cholangiopancreatography; ERBD, endoscopic retrograde biliary drainage.

*Includes choledochojejunostomy and hepaticojejunostomy.

surgery, and choledocholithotomy with T-tube insertion reached 82.3\%, 55.2\%, and 42.1\%, respectively. The median follow-up duration after complete resolution was 46.5 months (interquartile range, 21.6 to 76.7 months). None of the patients who maintained complete resolution by the last follow-up day developed CCA. Of the 43 patients with stone recurrence during follow-up, final resolution was achieved in seven patients; four patients by PTCSL, one by additional hepatectomy, one by biliary bypass surgery, and one by choledocholithotomy with T-tube insertion. CCA did not occur in patients who achieved final resolution, while three of the 40 patients (7.5\%) with stone recurrence developed CCA. Meanwhile, in 18 patients with residual stones after surgical therapy, three patients achieved final resolution by PTCSL, and none of them developed CCA. Among 15 patients with persistent residual stones, two patients (13.3\%) developed CCA. There was no significant difference in the CCA incidence between patients with stone recurrence after resolution and patients with persistent residual stones $(\mathrm{p}=0.886)$.

\section{Risk factors for CCA}

Except for patients who underwent radical hepatectomy within 6 months after initial diagnosis, the baseline demographics, imaging findings, and clinical data of 234 patients were analyzed to identify factors associated with CCA (Table 3). In 22 patients with CCA, the tumor was most commonly located in

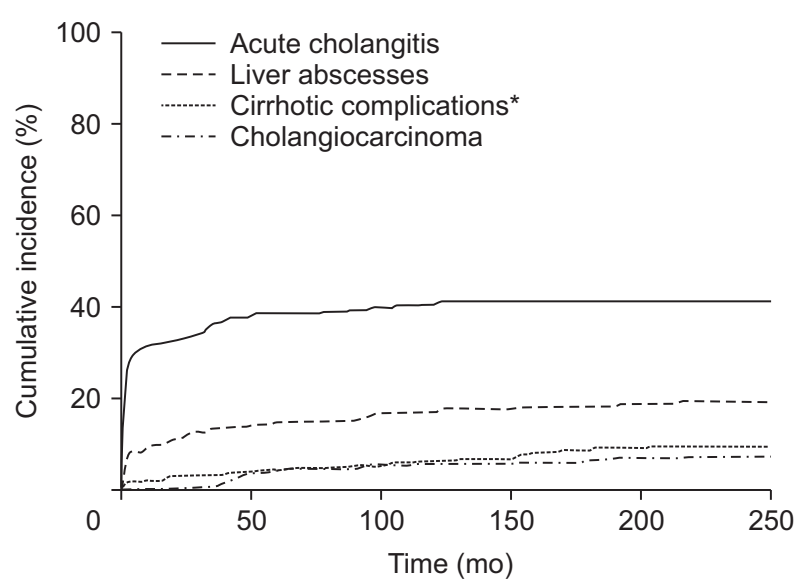

Fig. 2. Cumulative incidences of acute cholangitis, liver abscesses, cirrhotic complications, and cholangiocarcinoma in recurrent pyogenic cholangitis (RPC) patients. *Includes portal hypertensive complications related to biliary cirrhosis including varices, ascites, and portosystemic encephalopathy.

a left lobe in 10 patients (47.8\%), followed by the right lobe in eight patients (34.8\%), both lobes in three patients (13.0\%), and the mid-CBD in one patient (4.3\%).

All patients who had CCA in both lobes also exhibited leftsided atrophy, whereas 10 patients with left-sided CCA showed variations in atrophy; seven (70.0\%) showed atrophy on the left, one $(10.0 \%)$ showed atrophy on the right, one (10.0\%) showed atrophy on both sides, and one did not show liver atrophy. Among eight patients with right-sided CCA, atrophy was located in both lobes in four patients (50\%), in the right lobe in three patients (37.5\%), and in the left lobe in one patient (12.5\%). The patients with mid-CBD cancer experienced recurrent episodes of cholangitis due to the stricture of the involved site. In total, 21 patients developed intrahepatic CCA, with a total of 24 liver lobes containing CCA. Of these, 16 lobes (66.7\%) were affected by liver atrophy, whereas CCA developed in eight lobes (33.3\%) without atrophy ( $p=0.023)$.

In the multivariate analysis, bilateral atrophy significantly increased the risk of CCA (HR, 4.56; 95\% CI, 1.48 to 14.09 ; $\mathrm{p}=0.008$ ), while bilateral IHD stones and biliary cirrhosis were not associated with CCA development (HR, 2.03; 95\% CI, 0.86 to 4.79 ; $\mathrm{p}=0.106$ and HR, 0.82; 95\% CI, 0.26 to $2.52 ; \mathrm{p}=0.724$, respectively). In the subgroup analysis of patients with initial liver atrophy, the association of concomitant IHD stones and bile duct strictures or dilatation with the CCA-free probability was evaluated (Fig. 3). The log-rank test showed that IHD stones accompanied by atrophy had a tendency to increase the risk of CCA development, but this increase was not statistically significant $(p=0.189)$.

\section{DISCUSSION}

The main purpose of this research was to examine the natural course of RPC and identify risk factors for CCA. In this study, acute cholangitis requiring hospitalization occurred in $41.3 \%$ of 
Table 3. Univariate and Multivariate Analysis of Potential Factors for Cholangiocarcinoma

\begin{tabular}{|c|c|c|c|c|c|c|}
\hline \multirow{2}{*}{ Variable } & \multirow{2}{*}{$\begin{array}{c}\text { No. } \\
(\mathrm{n}=234)\end{array}$} & \multirow{2}{*}{$\begin{array}{c}\text { CCA } \\
\text { occurrence (\%) }\end{array}$} & \multicolumn{2}{|c|}{ Univariate analysis } & \multicolumn{2}{|c|}{ Multivariate analysis } \\
\hline & & & HR $(95 \%$ CI) & $\mathrm{p}$-value & HR $(95 \%$ CI) & p-value \\
\hline \multicolumn{7}{|l|}{ Age $\geq 65$ yr } \\
\hline No & 157 & $19(12.1)$ & 1.00 & & & \\
\hline Yes & 77 & $3(3.9)$ & $0.69(0.20-2.37)$ & 0.550 & $0.71(0.18-2.75)$ & 0.620 \\
\hline \multicolumn{7}{|l|}{ Sex } \\
\hline Male & 74 & $5(6.8)$ & 1.00 & & & \\
\hline Female & 160 & 17 (10.6) & $2.01(0.73-5.48)$ & 0.175 & $1.57(0.50-4.95)$ & 0.438 \\
\hline \multicolumn{7}{|l|}{ IHD stone } \\
\hline No & 56 & $3(5.4)$ & 1.00 & & & \\
\hline Both & 55 & $9(16.4)$ & $2.36(0.64-8.74)$ & 0.198 & $2.03(0.86-4.79)$ & 0.106 \\
\hline Left & 83 & $8(9.6)$ & $1.58(0.42-6.04)$ & 0.500 & $0.87(0.18-4.16)$ & 0.860 \\
\hline Right & 40 & $2(5.0)$ & $0.71(0.12-4.27)$ & 0.711 & $0.46(0.06-3.51)$ & 0.453 \\
\hline \multicolumn{7}{|c|}{ IHD dilatation or stricture } \\
\hline No & 8 & $1(16.7)$ & 1.00 & & & \\
\hline Both & 128 & $11(8.6)$ & $1.20(0.15-9.45)$ & 0.860 & $1.59(0.37-6.82)$ & 0.876 \\
\hline Left & 66 & $6(9.1)$ & $1.21(0.14-10.38)$ & 0.863 & $0.87(0.18-4.16)$ & 0.716 \\
\hline Right & 32 & $4(12.6)$ & $1.14(0.13-10.29)$ & 0.910 & $3.27(0.31-34.80)$ & 0.327 \\
\hline \multicolumn{7}{|l|}{ Liver atrophy } \\
\hline No & 99 & $5(5.1)$ & 1.00 & & & \\
\hline Both & 23 & $5(21.7)$ & $6.13(1.72-21.79)$ & 0.005 & $4.56(1.48-14.09)$ & 0.008 \\
\hline Left & 79 & $9(11.4)$ & $2.77(0.93-8.28)$ & 0.068 & $2.26(0.87-5.90)$ & 0.095 \\
\hline Right & 33 & $3(9.1)$ & $3.35(0.74-15.16)$ & 0.116 & $2.23(0.52-9.55)$ & 0.281 \\
\hline \multicolumn{7}{|c|}{ Previous hepatic resection } \\
\hline No & 206 & $19(9.2)$ & 1.00 & & & \\
\hline Yes & 28 & $3(10.7)$ & $1.06(0.31-3.61)$ & 0.921 & $1.58(0.42-6.03)$ & 0.500 \\
\hline \multicolumn{7}{|c|}{ Previous biliary bypass surgery* } \\
\hline No & 207 & $21(10.1)$ & 1.00 & & & \\
\hline Yes & 27 & $1(3.7)$ & $0.28(0.04-2.09)$ & 0.215 & $0.32(0.04-2.60)$ & 0.284 \\
\hline \multicolumn{7}{|l|}{ Acute cholangitis } \\
\hline No & 136 & $15(11.0)$ & 1.00 & & & \\
\hline Yes & 98 & $7(7.1)$ & $0.67(0.27-1.64)$ & 0.381 & $0.97(0.36-2.58)$ & 0.943 \\
\hline \multicolumn{7}{|l|}{ Liver abscess } \\
\hline No & 186 & $17(9.1)$ & 1.00 & & & \\
\hline Yes & 48 & $5(10.4)$ & $1.09(0.40-2.98)$ & 0.860 & $1.45(0.46-4.54)$ & 0.527 \\
\hline \multicolumn{7}{|l|}{ Biliary cirrhosis } \\
\hline No & 188 & $17(9.0)$ & 1.00 & & & \\
\hline Yes & 46 & $5(10.9)$ & $0.96(0.35-2.63)$ & 0.941 & $0.82(0.26-2.52)$ & 0.724 \\
\hline
\end{tabular}

CCA, cholangiocarcinoma; HR, hazard ratio; CI, confidence interval; IHD, intrahepatic duct.

*Includes choledochojejunostomy and hepaticojejunostomy.

the patients, and chronic complications included liver abscesses, biliary cirrhosis, and CCA in 19.4\%, 9.7\%, and 7.4\% of the patients, respectively. In particular, the risk of CCA increased in patients with liver atrophy, and the tumors were located mainly in atrophied lobes. Among surgical methods, hepatectomy was most commonly implemented, and CCA did not occur in patients who maintained complete remission after surgery. Although the occurrence of RPC is declining, ${ }^{14}$ RPC has clinical importance in that it is diagnosed at a relatively young age and is often accompanied by chronic complications. Periductal inflammation induces portal thrombosis, resulting in progressive liver atrophy, which may lead to a cirrhotic appearance of the liver. ${ }^{5,15}$ It is generally known that the risk of CCA is increased in RPC patients. ${ }^{4,16,17}$

The incidence of acute cholangitis in patients with RPC rang- 
A

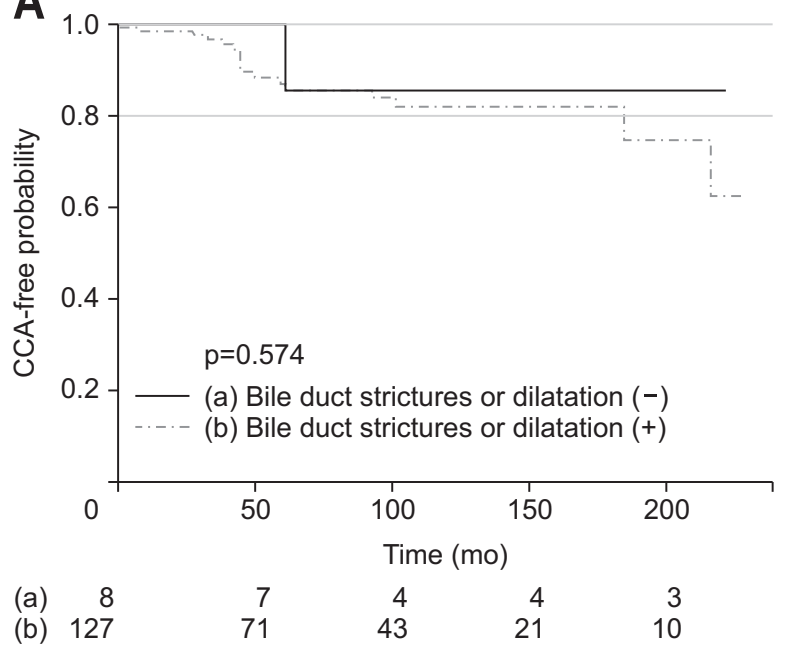

B

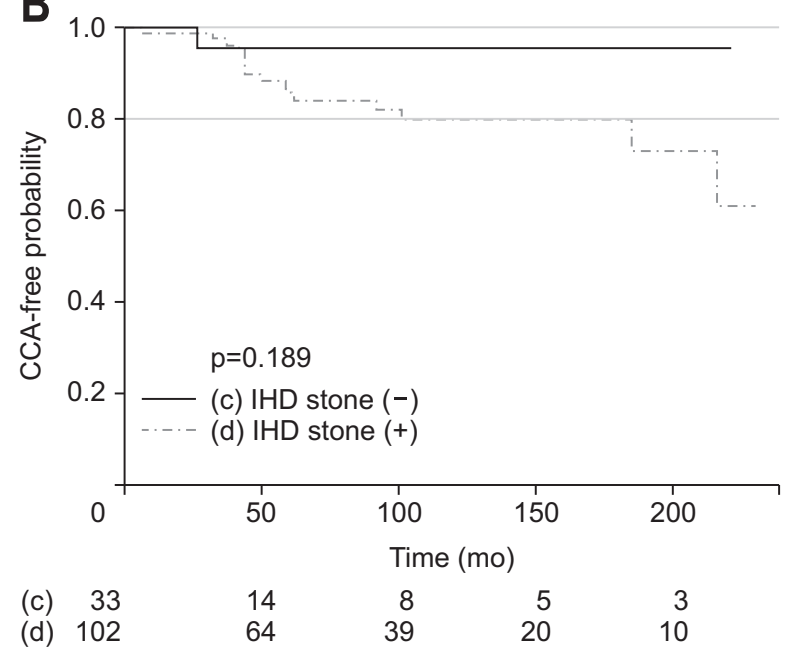

Fig. 3. Kaplan-Meier curves for cholangiocarcinoma (CCA)-free course and number of patients at risk among subgroups of patients with initial liver atrophy, classified by bile duct strictures or dilatation (A) and an intrahepatic duct (IHD) stone (B).

es from $40 \%$ to $100 \%$, which coincides well with this study. ${ }^{4,717}$ Likewise, the incidence of liver abscesses in this study is similar to that in a previous study. ${ }^{9}$ As cholangitis and liver abscesses often lead to life-threatening complications, such as septic shock, urgent intervention and antibiotic treatment are essential. ${ }^{18}$ The incidence rate of biliary cirrhosis in this study was relatively high compared with that in a previous study, which showed a $6.0 \%$ incidence rate for biliary cirrhosis during follow-up. ${ }^{12}$ This difference may be due to differences in the length of the follow-up duration. In our study, among patients who developed biliary cirrhosis, more than half of the patients showed moderate to severe liver function compromise (either Child-Pugh class B or C). Since progressive cirrhosis may lead to liver failure and eventually death, it is important to observe RPC patients closely with periodic examinations to determine whether there are clinical findings suggestive of cirrhosis or acute inflammatory complications.

Liver cirrhosis is known as an important risk factor for CCA. In our study, however, the final multivariate analysis revealed only bilateral atrophy to be related to an increased risk of CCA. This inconsistency may stem from the different inclusion criteria; previous studies have mainly included patients with cirrhosis caused by viral or alcoholic hepatitis other than biliary cirrhosis. ${ }^{19,20} \mathrm{~A}$ recent study based on the pathological findings of RPC patients showed that hepatic parenchyma outside the atrophic area was relatively normal in RPC-related biliary cirrhosis unlike in liver cirrhosis due to other causes; the patterns of ductal reaction and cholestasis were also different. ${ }^{21}$ The destruction and fibrosis of the hepatic parenchyma due to RPC-related atrophy rather than secondary cirrhotic change with portal hypertension seem to be related to the occurrence of CCA.

Kim et al..$^{22}$ have reported that $88 \%$ of CCA tumors are predominantly located in atrophied liver in patients with RPC. The results of our study are generally in accordance with those of this investigation, and CCA did not occur in patients who un- derwent surgical treatment and maintained complete remission, which suggests that periodic surveillance and early intervention are essential in high-risk patients. However, there are difficulties in treating patients with bilateral atrophy, and definitive treatment guidelines for such cases do not exist. ${ }^{23}$ Yang et al. ${ }^{24}$ have reported that bilateral hepatectomy and left-sided hepatectomy with concomitant PTCSL for right-side IHD stones are effective for reducing the long-term stone recurrence rate. However, if accompanied by extensive liver atrophy, optimal management remains very difficult and challenging. To decrease perioperative complications and improve long-term outcomes, a tailored approach predicting the remaining liver volume and liver function is necessary.

In this study, the diagnosis of RPC was based on characteristic imaging findings, including IHD dilatation with decreased branching and abrupt tapering, stenosis or stricture of peripheral ducts, and centrally located segmental ductal strictures with or without bile duct stones. ${ }^{9}$ The inclusion criteria in this study differ from other studies that included clinical symptoms for the scope of the diagnosis. ${ }^{6,8,10}$ On the other hand, several studies have used primary hepatolithiasis and RPC interchangeably., ${ }^{3,421}$ There have been dissimilarities in the definition of RPC in previous studies regarding the presence of IHD stones or typical symptoms indicating cholangitis. ${ }^{4,7,10,25}$ Collectively, the diagnostic criteria for RPC are heterogeneous, and standard diagnostic criteria have not yet been established. Meanwhile, a recent study comparing the pathological findings of Asian RPC patients with those of Western patients with a benign biliary stricture concluded that the pathological findings of both diseases are almost identical, ${ }^{21}$ suggesting that the diagnostic spectrum of RPC may overlap with that of a benign biliary stricture. Conclusively, the diagnostic range and nomenclature for RPC should be discussed actively, and efforts to clarify the pathogenesis of RPC are needed.

This study has limitations in terms of its single-center retro- 
spective design. Since there are no standardized routine tests for the initial diagnosis or follow-up protocols for RPC, baseline etiological evaluations, including for clonorchiasis, a well-known risk factor for RPC, were not performed uniformly. In addition, patients were followed at different time intervals and underwent examination with different imaging modalities, which may present differences in the sensitivity of detecting RPC-related complications. Second, the sample size of CCA patients was relatively small to perform an adequately powered analysis, which can lead to statistical biases. Third, since there was no validation group in this study, our findings need to be validated further in a larger independent cohort of patients with RPC. Nevertheless, this study is the first to elucidate the natural course of RPC and evaluate factors associated with CCA based on long-term follow-up data.

In conclusion, RPC is a progressive disease with several chronic complications, including acute cholangitis, liver abscesses, biliary cirrhosis, and CCA. Active surgical measures should be implemented in patients with high-risk factors, such as liver atrophy, to prevent CCA.

\section{CONFLICTS OF INTEREST}

No potential conflict of interest relevant to this article was reported.

\section{ACKNOWLEDGEMENTS}

Author contributions: Conception and design: S.H.L. Collection and assembly of data: Y.H.C., J.H.C., B.S.S., G.H. Data analysis and interpretation: J.K., D.K.J., W.H.P. Extensive review and revision of manuscript: J.K.R., Y.T.K., J.K.L. Manuscript writing: M.S.Y. All authors read and approved the final manuscript.

\section{REFERENCES}

1. Digby KH. Common-duct stone of liver origin. Br J Surg 1930:17:578-591.

2. Cook J, Hou PC, Ho HC, Mcfadzean AJ. Recurrent pyogenic cholangeitis. Br J Surg 1954;42:188-203.

3. Tsui WM, Lam PW, Lee WK, Chan YK. Primary hepatolithiasis, recurrent pyogenic cholangitis, and oriental cholangiohepatitis: a tale of 3 countries. Adv Anat Pathol 2011;18:318-328.

4. Lee KF, Chong CN, Ng D, et al. Outcome of surgical treatment for recurrent pyogenic cholangitis: a single-centre study. HPB (Oxford) 2009;11:75-80.

5. Kwan KE, Shelat VG, Tan CH. Recurrent pyogenic cholangitis: a review of imaging findings and clinical management. Abdom Radiol (NY) 2017;42:46-56.

6. Law ST, Li KK. Is pyogenic liver abscess associated with recurrent pyogenic cholangitis a distinct clinical entity? A retrospective analysis over a 10-year period in a regional hospital. Eur J Gastroenterol Hepatol 2011;23:770-777.
7. Ray S, Sanyal S, Das K, et al. Outcome of surgery for recurrent pyogenic cholangitis: a single center experience. HPB (Oxford) 2016;18:821-826.

8. Co M, Pang SY, Wong KY, Ip WK, Yuen WK. Surgical management of recurrent pyogenic cholangitis: 10 years of experience in a tertiary referral centre in Hong Kong. HPB (Oxford) 2014;16:776-780.

9. Kim MJ, Cha SW, Mitchell DG, Chung JJ, Park S, Chung JB. MR imaging findings in recurrent pyogenic cholangitis. AJR Am J Roentgenol 1999;173:1545-1549.

10. Koh YX, Chiow AK, Chok AY, Lee LS, Tan SS, Ibrahim S. Recurrent pyogenic cholangitis: disease characteristics and patterns of recurrence. ISRN Surg 2013;2013:536081.

11. Cheon YK, Cho YD, Moon JH, Lee JS, Shim CS. Evaluation of long-term results and recurrent factors after operative and nonoperative treatment for hepatolithiasis. Surgery 2009;146:843-853.

12. Venkatesh SK, Chandan V, Roberts LR. Liver masses: a clinical, radiologic, and pathologic perspective. Clin Gastroenterol Hepatol 2014;12:1414-1429.

13. Venables WN, Ripley B. Modern applied statistics with S. 4th ed. New York: Springer, 2002.

14. Lo CM, Fan ST, Wong J. The changing epidemiology of recurrent pyogenic cholangitis. Hong Kong Med J 1997;3:302-304.

15. Kusano S, Okada Y, Endo T, Yokoyama H, Ohmiya H, Atari H. Oriental cholangiohepatitis: correlation between portal vein occlusion and hepatic atrophy. AJR Am J Roentgenol 1992;158:1011-1014.

16. Chen DW, Tung-Ping Poon R, Liu CL, Fan ST, Wong J. Immediate and long-term outcomes of hepatectomy for hepatolithiasis. Surgery 2004;135:386-393.

17. Al-Sukhni W, Gallinger S, Pratzer A, et al. Recurrent pyogenic cholangitis with hepatolithiasis: the role of surgical therapy in North America. J Gastrointest Surg 2008;12:496-503.

18. Heffernan EJ, Geoghegan T, Munk PL, Ho SG, Harris AC. Recurrent pyogenic cholangitis: from imaging to intervention. AJR Am J Roentgenol 2009;192:W28-W35.

19. Lee TY, Lee SS, Jung SW, et al. Hepatitis B virus infection and intrahepatic cholangiocarcinoma in Korea: a case-control study. Am J Gastroenterol 2008;103:1716-1720.

20. Tyson GL, El-Serag HB. Risk factors for cholangiocarcinoma. Hepatology 2011;54:173-184.

21. Zhao L, Hosseini M, Wilcox R, et al. Segmental cholangiectasia clinically worrisome for cholangiocarcinoma: comparison with recurrent pyogenic cholangitis. Hum Pathol 2015;46:426-433.

22. Kim JH, Kim TK, Eun HW, et al. CT findings of cholangiocarcinoma associated with recurrent pyogenic cholangitis. AJR Am J Roentgenol 2006;187:1571-1577.

23. Parray FQ, Wani MA, Wani NA. Oriental cholangiohepatitis: is our surgery appropriate? Int J Surg 2014;12:789-793.

24. Yang T, Lau WY, Lai EC, et al. Hepatectomy for bilateral primary hepatolithiasis: a cohort study. Ann Surg 2010;251:84-90.

25. Lim JH, Ko YT, Lee DH, Hong KS. Oriental cholangiohepatitis: sonographic findings in 48 cases. AJR Am J Roentgenol 1990;155:511-514. 